\title{
Childhood osteomyelitis: imaging characteristics
}

\author{
Joost van Schuppen • Martine M. A. C. van Doorn • \\ Rick R. van Rijn
}

Received: 11 January 2012 / Accepted: 5 July 2012 /Published online: 10 August 2012

(C) The Author(s) 2012. This article is published with open access at Springerlink.com

\begin{abstract}
Background The purpose of this review is to illustrate the imaging findings of childhood osteomyelitis. The diagnosis of childhood osteomyelitis can be challenging. Clinical presentation and laboratory results can differ and are relatively unreliable. To date, its role in the assessment of treatment efficacy is not yet clear.

Methods This review article provides an overview of the different imaging modalities and imaging characteristics of childhood osteomyelitis. Levels of evidence for different modalities are presented.

Results Paediatric radiology plays a pivotal role in the diagnosis of childhood osteomyelitis and can also be used to guide therapy and intervention.

Conclusion Although imaging is essential in the diagnostic process, cooperation between the physician and radiologist remains the cornerstone in accurately diagnosing childhood osteomyelitis.

Main Messages

- Imaging plays a pivotal role in the diagnosis of childhood osteomyelitis.

- Cooperation between the clinician and radiologist is a very important aspect of making the diagnosis.

- The initial imaging modality in childhood osteomyelitis is conventional imaging.

- Normal conventional imaging does not exclude osteomyelitis.

J. van Schuppen · M. M. A. C. van Doorn · R. R. van Rijn Department of Radiology, Academic Medical Center/Emma Children's Hospital,

Amsterdam, The Netherlands

J. van Schuppen $(\square)$

Department of Radiology, Academic Medical Center/Emma

Children's Hospital,

Meibergdreef 9,

1105 AZ Amsterdam Zuid-Oost, The Netherlands

e-mail: j.vanschuppen@amc.uva.nl
\end{abstract}

Keywords Paediatrics · Musculoskeletal diseases · Osteomyelitis $\cdot$ Radiology $\cdot$ Diagnostic imaging

$\begin{array}{ll}\text { Abbreviations } & \\ \text { AHOM } & \text { Acute haematogenous osteomyelitis } \\ \text { ALARA } & \text { As low as reasonably achievable } \\ \text { COM } & \text { Chronic osteomyelitis } \\ \text { CRMO } & \begin{array}{l}\text { Chronic recurrent } \\ \text { multifocal osteomyelitis }\end{array} \\ \text { CRP } & \text { C-reactive protein } \\ \text { ESR } & \text { Erythrocyte sedimentation rate } \\ \text { eGFR } & \text { Estimated glomerular filtration } \\ & \text { rate (ml/min) } \\ \text { FDG-PET } & \text { Fluorodeoxyglucose (18F) } \\ & \text { positron emission tomography } \\ \text { FS } & \text { Fat saturation } \\ \text { MRI } & \text { Magnetic resonance imaging } \\ \text { MRSA } & \text { Methycillin-resistant } \\ \text { NSF } & \text { Staphylococcus areus } \\ \text { PD } & \text { Nephrogenic systemic fibrosis } \\ \text { SAPHO syndrome } & \text { Proton density } \\ & \text { Synovitis, acne, pustulosis, } \\ \text { hy } & \text { hyperostosis, osteitis syndrome } \\ \text { STIR } & \text { Spectral presaturation } \\ \text { TSE } & \text { inversion recovery } \\ \text { US } & \text { Short T1/tau inversion recovery } \\ & \text { Turbo spin echo } \\ \text { Ultrasound/ultrasonography }\end{array}$

\section{Introduction}

Childhood osteomyelitis can be a challenging problem to both the clinician and the radiologist as the clinical presentation can be variable in severity, and laboratory results are relatively unsupportive in the diagnostic workup. Radiology plays a pivotal role in its diagnosis and can also be used to 
guide therapy and intervention. This review discusses the pathophysiology of childhood osteomyelitis and the clinical presentation. With respect to radiological imaging, it will focus on the application of radiological techniques in the diagnosis of childhood osteomyelitis and the reported levels of evidence for the use of these techniques (Table 1).

\section{Osteomyelitis}

Pathophysiology

Osteomyelitis is defined as an infection of the bone, bone marrow and surrounding soft tissue. In childhood the most common route of infection is haematogenous spread of a microorganism. Secondary spread by contiguity and direct spread of infection, for example due to direct penetration e.g. in cases of comminuted fractures, are seen less often [2, 3]. Osteomyelitis can also follow after trauma, where a metaphysical haematoma can function as a focus of infection [4]. Osteomyelitis as a result of vascular insufficiency, e.g. as commonly seen in adult diabetic patients, is seldom seen in childhood and will therefore not be discussed in this review.

In order to understand the clinical and radiological findings in childhood osteomyelitis, it is important to recall the anatomy of the paediatric skeleton. In growing bone the diaphysis and metaphysis share the same nutrient arteries and veins. These arteries and veins form a fine network of arterioles and venules in the metaphysis, which leads to the formation of so-called sinusoidal lakes. These sinusoidal lakes can act as pools where microorganisms can accumulate, thus leading to a focus of osteomyelitis. In contrast to the adult situation, in young children the epiphysis has its own nutrient vessels. After 12 to 18 months of age, these transphysial vessels disappear. As a result the physis acts as a natural border and prevents the spread of osteomyelitis from the metaphysis to the epiphysis. Spread to the epiphysis and joints is less common.

Neonates are more prone to osteomyelitis. Because of a less developed immune system, osteomyelitis can be caused by less virulent agents and tends to present fewer clinical signs. The combination of unclear symptoms in neonates and the presence of transphysial vessels can lead to indolent infections that are often discovered at a late stage $[3,4]$.
The reported annual incidence of childhood osteomyelitis is 3 to 20 per 100,000. For acute osteomyelitis the incidence is 8 per 100,000 and for sub-acute osteomyelitis 5 per 100,000 $[2,3,5,6]$. The incidence is higher in children below 3 years of age, with a peak incidence in children below 1 year of age. Acute osteomyelitis occurs more often in boys, with a reported male-female ratio of 1.9 to $1.0[2,3,5-7]$.

The most common site of infection is the long bones, especially the femur and tibia. Most infections are monoostotic, but polyostotic involvement of up to $6.8 \%$ is reported in infants, and even $22 \%$ in neonates [6].

Risk factors for osteomyelitis include trauma, sickle cell disease, immunodeficiency, sepsis, minor trauma in combination with bacteraemia, an indwelling vascular catheter and chronic vascular lines, for example in case of haemodialysis $[4,7]$.

The differential diagnoses based on radiological features for acute and chronic osteomyelitis are summarised in Table 2.

\section{Clinical findings and laboratory tests}

Clinical presentation can be diverse and therefore confusing. Usually pain and reluctance to move limbs are present. Also fever, swelling and tenderness can be present. Acute haematogenous osteomyelitis (AHOM) is defined as the presence of complaints for fewer than 14 days, whereas the subacute form persists longer than 14 days.

In osteomyelitis with anaerobic organisms, even the presentation can be without symptoms or just mild systemic symptoms such as low fever can be present [8].

A study by Riise et al. showed that an erythrocyte sedimentation rate (ESR) of more than $40 \mathrm{~mm} / \mathrm{h}$ has the highest predictive value (26\%) [6]. All other tests had a lower predictive value. Blood cultures were only positive in $26 \%$ of cases of acute osteomyelitis and negative in $100 \%$ of cases of sub-acute osteomyelitis. Other authors report a higher percentage of positive blood tests [9]. The white blood cell count can be normal, whereas C-reactive protein (CRP) and ESR levels are raised in most cases [6].

The most common organism causing acute haematogenic osteomyelitis is Staphylococcus aureus (up to $95 \%$ ), followed by $\beta$-haemolytic Streptococcus, Streptococcus pneumoniae, Escheria coli and Pseudomonas aeruginosa.
Table 1 Classification of levels of evidence used in this review [18]

\begin{tabular}{ll}
\hline Level & Description \\
\hline Level I & $\begin{array}{c}\text { Strong evidence based on studies with a broad generalisability or meta-analyses based on level I studies } \\
\text { Level II } \\
\text { Moderate evidence based on prospective or retrospective studies with narrow generalisability or } \\
\text { cohort studies, case control studies or randomised control trials }\end{array}$ \\
Level & Limited evidence based on diagnostic accuracy studies with several flaws in research methods or \\
III & on nonrandomised comparison studies based on outcomes \\
Level & $\begin{array}{c}\text { Insufficient evidence, studies with multiple flaws in research methods or case series, descriptive } \\
\text { IV }\end{array}$ \\
\hline
\end{tabular}


Table 2 Differential diagnosis of acute and chronic osteomyelitis in children

\begin{tabular}{|c|c|}
\hline Differential diagnosis & Major imaging or clinical feature \\
\hline \multicolumn{2}{|l|}{ Acute osteomyelitis } \\
\hline Vaso-occlusive disease & $\begin{array}{l}\text { Linear hypointense on } \mathrm{T} 1 \text { - and } \mathrm{T} 2 \text {-weighted changes in meta- and } \\
\text { epiphysis }\end{array}$ \\
\hline Septic emboli & Growth plate involvement in fulminant meningococcemia \\
\hline Septic arthritis & Fluid in joints \\
\hline Spondylodiscitis & $\begin{array}{l}\text { Imaging shows low signal of the disc with fluid/abscess around it } \\
\text { with destruction of the vertebrae, rim enhancement after } \\
\text { gadolinium. (image 9) }\end{array}$ \\
\hline Osteoid osteoma & Cortical sclerotic lesion with typical lucent nidus \\
\hline ALL & Diffuse bone marrow changes, $\mathrm{T} 1$ low signal and $\mathrm{T} 2$ heterogeneous \\
\hline Stress fracture & $\begin{array}{l}\text { Linear lesions show hypointense changes on T1, without } \\
\text { enhancement }\end{array}$ \\
\hline Metastastic neuroblastoma & Multiple lesions with high signal on STIR. In context of neuroblastoma \\
\hline Ewing's sarcoma & Large soft tissue mass, onion-skin periostitis, metastasis \\
\hline Osteosarcoma & Codman's triangle, sunburst spiculated periostitis, cortical destruction \\
\hline $\begin{array}{l}\text { Self-limiting sternal tumours of } \\
\text { childhood (SELSTOC) }\end{array}$ & $\begin{array}{l}\text { Ultrasound shows dumbbell-shaped lesions extending to the area } \\
\text { behind the sternal bone, involving the cartilage, leading to in- } \\
\text { creased distance between ossification centres }\end{array}$ \\
\hline \multicolumn{2}{|l|}{ Chronic osteomyelitis } \\
\hline Ewing’s sarcoma & Large soft tissue mass, onion-skin periostitis, metastasis \\
\hline $\mathrm{LCH}$ & $\begin{array}{l}\text { Typical punched-out lesion on conventional imaging. Whole-body } \\
\text { MRI STIR can be used for screening }\end{array}$ \\
\hline Metastasis & Multifocal lesions, no inflammation parameters \\
\hline CRMO & $\begin{array}{l}\text { STIR and T2 series show multiple spots of high signal intensity, and } \\
\text { series after contrast show enhancement. Imaging characteristics are } \\
\text { comparable with acute osteomyelitis. Focus of osteomyelitis and } \\
\text { symptoms can change over time. PET scan can also show multiple } \\
\text { sites of uptake }\end{array}$ \\
\hline
\end{tabular}

Besides these microorganisms, fungi, viruses and parasites have been reported to cause childhood osteomyelitis (Table 3) $[3,9]$.
In recent years the prevalence of methycillin-resistant Staphylococcus areus (MRSA) osteomyelitis has increased. MRSA leads to more aggressive cases of osteomyelitis, also
Table 3 Causative pathogens in childhood osteomyelitis $[10,16$, $24,26]$

${ }^{\mathrm{a}}$ Seen in endemic areas

\begin{tabular}{|c|c|}
\hline Causative pathogens & Incidence \\
\hline Staphylococcus aureus/MRSA & $30-95 \%$ \\
\hline Streptococcus pneumoniae & $0.5-17 \%$ \\
\hline Streptococcus pyogenes & $17 \%$ \\
\hline$\beta$-Haemolytic streptococcus & $0.5-6 \%$ \\
\hline Pseudomonas aeruginosa & $4.2 \%$ \\
\hline Group A Streptococcus bacterium & $4.2 \%$ \\
\hline Kingella kingae & $1.4 \%$ \\
\hline Escheria coli & $0-0.5 \%$ \\
\hline Candida albicans/Coccidioides immitis & $0.5 \%$ \\
\hline Aspergillus & Unknown \\
\hline Tuberculosis $^{\mathrm{a}}$ & Unknown \\
\hline Salmonella & In case of sickle cell disease \\
\hline Parasites $^{\mathrm{a}}$ & Unknown \\
\hline Anaerobic & Unknown[8] \\
\hline Unknown (no positive culture) & $25-38 \%$ \\
\hline
\end{tabular}


with a higher prevalence of abscess formation and other complications such as myositis and pyomyositis [11].

Specific pathogens strongly depend on age, the immune status of the patient and the geographic location for endemic diseases, such as tuberculosis [12].

Since the introduction of the Haemophilus influenza type $b$ vaccination in the national vaccination programs, the incidence of septic osteomyelitis and arthritis due to this organism has been significantly reduced. Nevertheless, this organism should be kept in mind as a causative agent in areas without a vaccination programme and also in possible cases of failure of the vaccine $[8,13]$.

In sickle cell disease the diagnosis of osteomyelitis can be more challenging, and in these patients clinical signs and laboratory testing can be misleading. Pain in joints and bones can be a sign of a sickle cell crisis/ osteonecrosis but also of osteomyelitis. Interpretation of imaging, especially MR, as discussed below, can be challenging when differentiating between osteomyelitis and sickle cell crisis/osteonecrosis [14].

\section{Complications and outcome}

A complication of osteomyelitis can be the development of an abscess, typically known as Brodie's abscess. Also the development of sequesters, fistulas and sinus tract lesions can be seen. Although CT and MR imaging is useful in delineating the extension of abnormalities, it is difficult to differentiate between an active or an inactive focus of osteomyelitis. In these cases, PET-CT, which has a high sensitivity for disease activity, might play a role [15]. Other reported complications are septic arthritis, slipped epiphysis, damage of the physis causing early closing of the physis and eventually leading to growth retardation, or angulation deformation in the long bones [16].

\section{Principles of treatment}

Ideally treatment is based on the isolation of the pathogen from the focus of infection or blood. If this is not possible, empirical treatment, consisting of intravenous antibiotics in haematogenous osteomyelitis, should be started. Once the pathogen is known, treatment should be changed if needed. This approach has been shown to be effective, resulting in a cure rate of over $95 \%$ [17]. In a systematic review, no significant difference was found between a short (less than 7 days) and a long (1 week or longer) course of intravenous antimicrobial therapy when the clinical cure rate at 6 months was the primary outcome variable [18].

Surgical intervention is used as an adjunct to antibacterial treatment. The aim of surgery should be to drain intraosseous abscesses, removal of sequestra and debridement of adjoining infectious foci. Interventional radiology plays a role in percutaneous drainage of soft-tissue abscesses. Duration of therapy depends on the effect of therapy and surgical intervention [16]. For the latest standard in osteomyelitis treatment, the reader is referred to Up-to-Date [7].

Poly-microbacterial infections give rise to more complications. Response to treatment is often monitored by ESR and CRP. Follow-up imaging may be used to evaluate complications [19].

\section{Imaging techniques}

Conventional imaging Conventional radiography is the initial modality of choice to evaluate osseous changes. In the majority of cases it will be the only imaging technique used in the diagnosis and treatment of childhood osteomyelitis.

The ease of access and the relatively low radiation dose make it an ideal imaging tool for skeletal pathologies. Also it is possible to exclude other pathologies such as malignancies and fractures. However, conventional imaging should not be used to exclude osteomyelitis in the first 10 days of symptoms $[4,20]$.

Karmazyn et al. advise performing at least two orthogonal views of the body part of interest [19]. Comparison with radiographs of the opposite limb is seldom useful, and therefore they should not be routinely obtained.

Conventional imaging has a reported sensitivity of $20-75 \%$ and a specificity of $75-83 \%$, but evidence is limited (level of evidence II-III) $[2-4,9]$.

Ultrasound (US) Ultrasonography, especially in children, is a very useful and versatile modality. It allows correlating the physical exam with US findings and comparing the affected side with the opposite side [3,21, 22]. It is important to use a linear high-frequency transducer.

US has a high reported sensitivity for detection of subperiostal abscesses and fluid collections [3, 22], also complications such as soft tissue abscess, sequesters, fistulas and

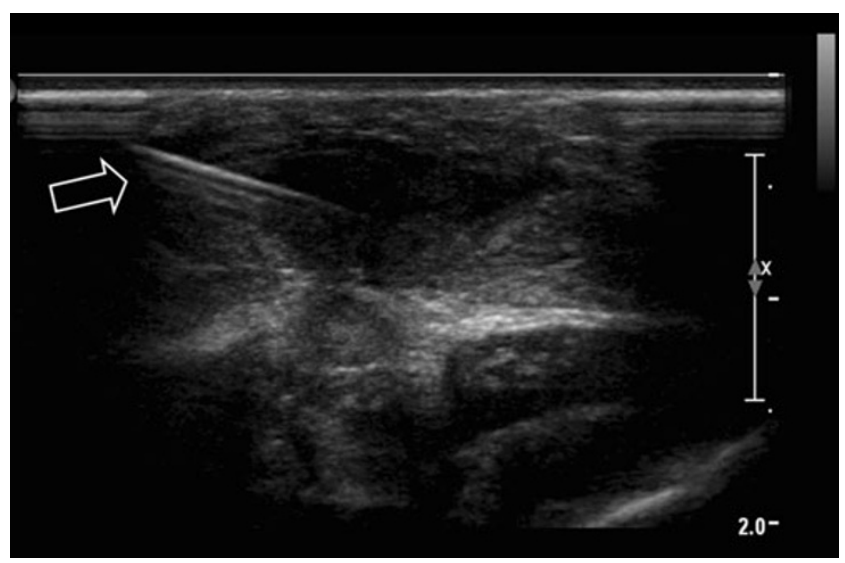

Fig. 1 Ultrasound-guided biopsie needle aspiration of a subcutaneous pre-sternal abscess 


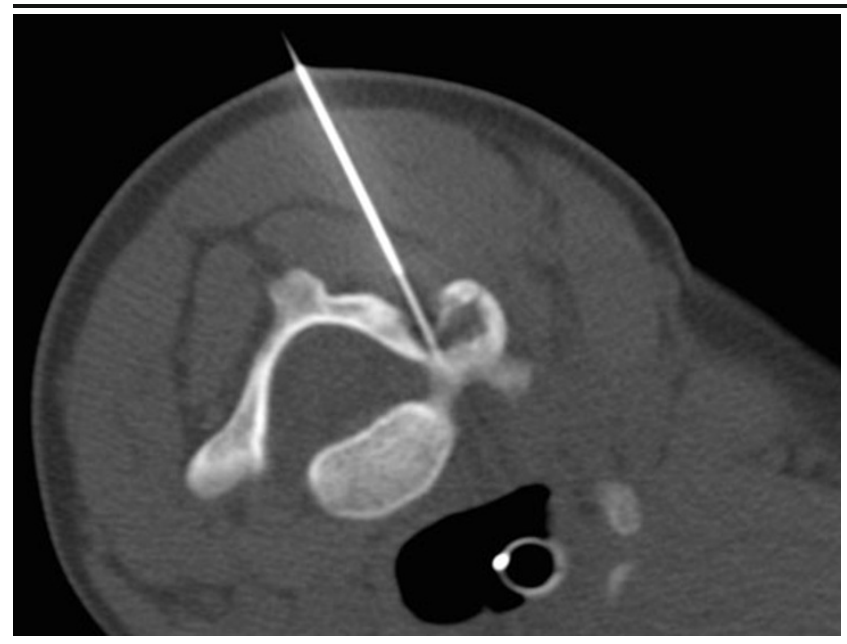

Fig. 2 A 15-month-old boy suspected to have spondylodiscitis. CT-guided needle aspiration was performed for analysis

sinus track formation can be evaluated by US. Furthermore, it is possible to perform direct intervention, such as aspiration or drainage of an abscess or joint effusion (Fig. 1). A drawback of US is the inability to evaluate bone marrow involvement and the operator dependency. The sensitivity of US in osteomyelitis is reported to be $46-74 \%$, with a high specificity of $63-100 \%$ (level of evidence: III) [21, 23].
Computed tomography (CT) In the acute workup of osteomyelitis, computed imaging plays no role.

In chronic osteomyelitis, it is more useful and superior to MR in detecting cortical destruction, air and sequesters [3, $4,19,20,24-26]$. The main advantage of $\mathrm{CT}$ is the high spatial resolution of bony structures and surrounding soft tissue, and can also be used in the diagnostic workup of CT for image-guided aspiration/intervention (Fig. 2).

A major disadvantage of $\mathrm{CT}$ is the relatively high amount of radiation; therefore, $\mathrm{CT}$ should be used according to the ALARA principle (As Low As Reasonably Achievable) [27].

No specific literature was found on the sensitivity and specificity of $\mathrm{CT}$ in detecting acute osteomyelitis and its complications. Based on a systematic review, the sensitivity and specificity of $\mathrm{CT}$ in chronic osteomyelitis were found to be respectively $67 \%$ and $50 \%$ (level of evidence: III) [28].

Magnetic resonance imaging (MRI) After conventional imaging MRI is the most important imaging modality for the evaluation of osteomyelitis. MRI not only allows excellent delineation of the osseous component but also soft tissue extension, joint effusion and complications of osteomyelitis such as abscess formation, especially in case of failure to respond to therapy [25].
Table 4 Sequences and indications

\author{
T1 SE/FSE \\ T2 FSE +/- FS \\ Contrast enhanced T1 FS \\ Delayed sequence \\ (3-10 minutes) \\ SE T1 pre/post injection \\ (subtraction image), \\ SE T1 FS \\ 3D GRE FS \\ Dynamic series \\ STIR
}

PD SE/FSE (FS)

Gradient Echo FS

DWI

Whole body MRI
Excellent sequence for illustration of anatomy, bone marrow (conversion), fat content, haemorrhage, calcifications, fracture line, tumor margins, soft tissue. It pairs a good image quality with a high spatial resolution

Sensitive for oedema, bone marrow, effusion and soft tissue (muscle oedema)

Fat suppression technique in combination with gadolinium, make it easier to see the enhancement

Contrast will change sensitivity and specificity and increases the confidence in making the diagnosis of osteomyelitis on MR.

High spatial resolution, or dynamic contrast series 3D GRE T1for functional imaging (perfusion) with high temporal resolution $(3-15 \mathrm{sec})$ and total acquisition time of 5 minutes

Are good for post-treatment evaluation. You should inject contrast agent if STIR/T2 and T1 sequences are normal.

Sensitive to oedema. It gives a homogenous fat saturation and the possibility of a large field of view. STIR sequences never after gadolinium injection, because the signal of gadolinium is suppressed

Perfect for anatomy, oedema. Also for evaluating meniscus, ligaments (SE $>$ FSE), articular cartilage, growth cartilage (zone of provisional calcification), and bone marrow (FS)

Excellent for cartilage, with 3DT1 or T2* is the best sequence for blood products

Perform $a b=0$ for the T2-shine-trough-effect. There is not enough evidence for the use of diffusion weighted imaging in osteomyelitis, although in the evaluation of osteomyelitis treatment this could have an application.

Evaluation of metastases/LCH, can be performed in case of multifocality 
In young children the main disadvantage of the use of MRI is the long scan time during which the patient has to remain immobile. This leads to the need for general anaesthesia (intubation) or sedation in young children. Other approaches, such as immobilisation with for example a vacuum mattress or a feed and swaddle protocol, have been advocated, thus obviating the need for general sedation [29]. For older children having enough time and patience to make the child feel comfortable and cooperative is mandatory. Adaptations to the environment, such as the use of an open MRI, the presence of parents or distraction during imaging, can also be helpful.

First of all, it's important to choose the appropriate coil for the maximum zone of interest and adequate details. There always needs to be a balance between the signal-to-noise ratio and image resolution. In children, it is wise to start with the most important sequences, in case the examination has to be stopped before the end of the planned scan. Long sequences should be avoided, but if really needed, they can be separated in contiguous series. The most important sequences for the diagnosis and follow-up of osteomyelitis are summarised in Table 4 [30]. The use of gadolinium-enhanced MR is still under debate, especially when unenhanced series are normal.
Nevertheless, research by Kan et al. shows that the sensitivity of contrast-enhanced MR is higher for abscesses than that of non-enhanced MR. Indications for using contrast are spinal osteomyelitis and cases where the initial studies are inconclusive and clinical symptoms persist $[4,31]$.

MRI has a high sensitivity for detection of osteomyelitis of $82-100 \%$ and high specificity of $75-99 \%$ (level of evidence: III) [3, 19, 24]. In cases of multifocal sites or if the localisation of the symptoms is doubtful, a whole-body MRI can be performed, although there is poor evidence (level of evidence: IV).

Nuclear imaging Bone scintigraphy can play a role when localisation of osteomyelitis, based on clinical information or other radiological methods, is not possible [32]. However, in the last few years nuclear imaging has increasingly been replaced by whole-body STIR MRI, thus allowing for one-stop-shop imaging.

The use of FDG-PET CT in the diagnosis of osteomyelitis has mainly been reported in adults [33]. For paediatric use a drawback is the high effective radiation dose, which can be in the range of 5-18 $\mathrm{mSv}$ [34].
Fig. 3 Flow chart of proposed imaging strategy

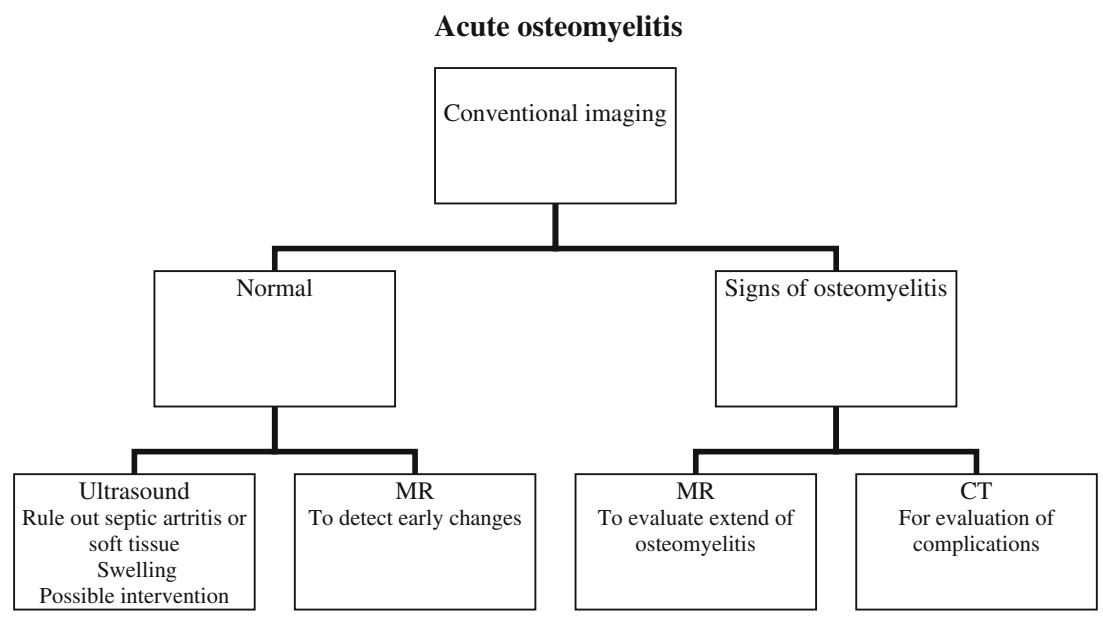

Chronic osteomyelitis

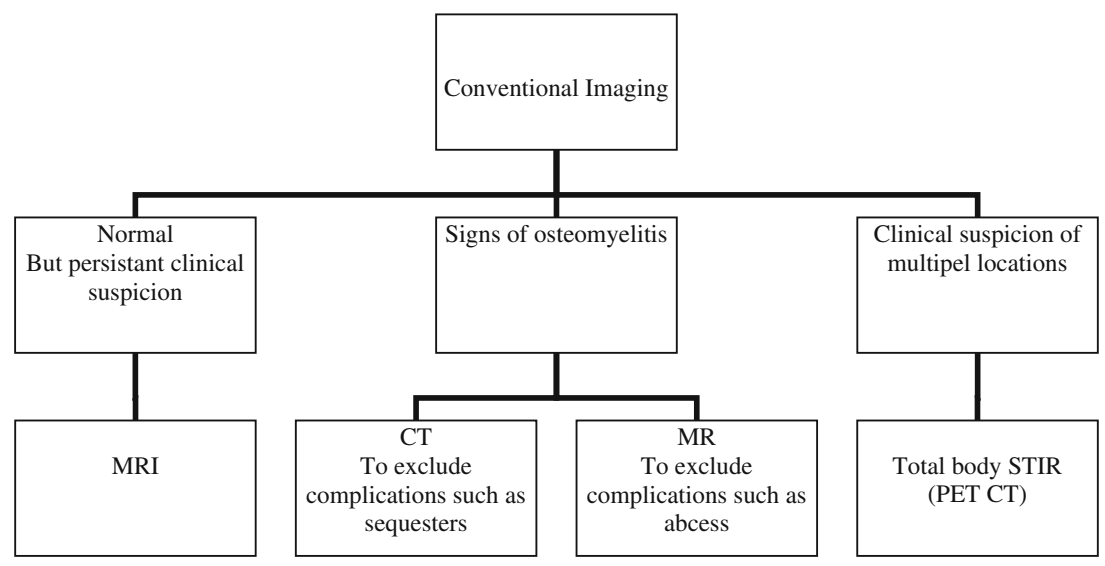


Although limited evidence is available on paediatric osteomyelitis, PET CT can effectively differentiate between an active and inactive focus of chronic osteomyelitis [19]. Literature on the use of FDG PET in adults shows a sensitivity of $94-100 \%$ and a specificity of $75-99 \%$ (level of evidence: IV) [19].

\section{Imaging findings in osteomyelitis}

\section{Strategy}

The different modalities and their advantages and disadvantages are discussed above. The imaging strategies for these modalities in the setting of acute and chronic osteomyelitis are summarised in a flowchart (Fig. 3).

\section{Acute osteomyelitis}

Acute haematogenous osteomyelitis is typically seen in young children and is clinically characterised by a rapid onset of complaints after contact with a pyogenic bloodborne organism. Its typical localisation is the metaphysis of the tibia and femur. Through the transphyseal nutrient

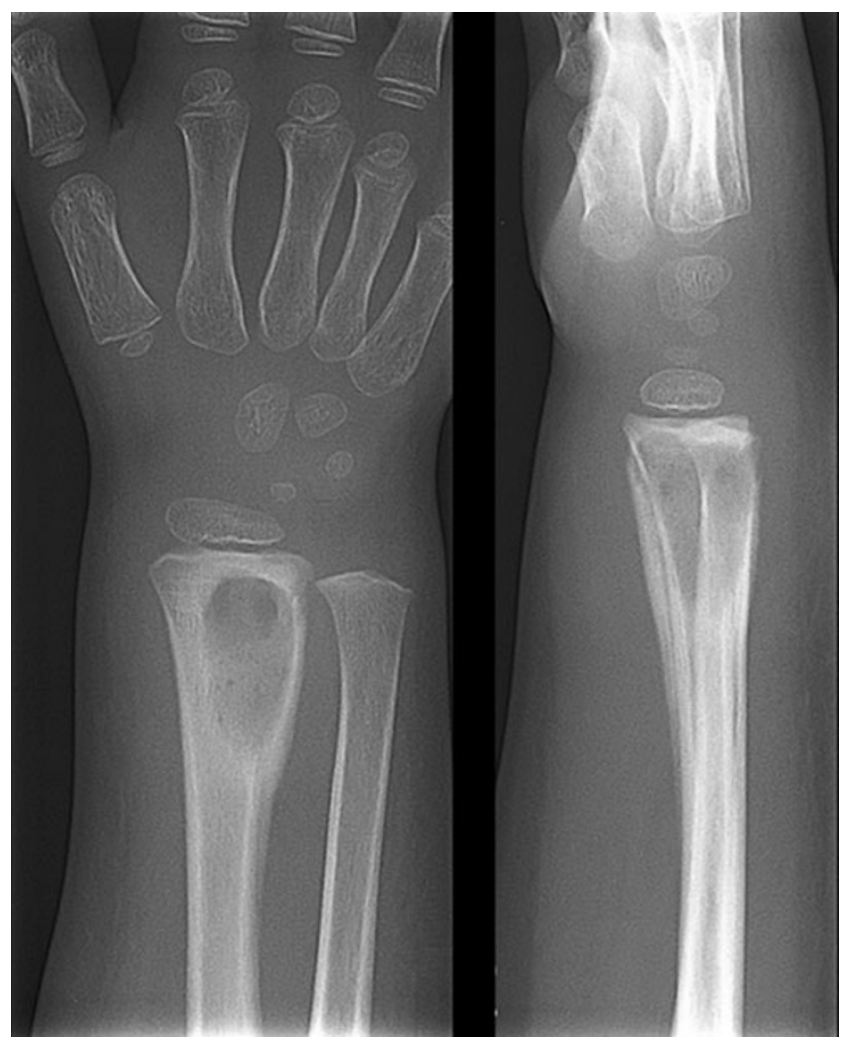

Fig. 4 A 3-year-old boy with osteomyelitis of the distal radius. Anterior-posterior and lateral conventional radiographs show a sharply delineated lucent lesion in the distal radius vessels, spreading to the physis, epiphysis and joints is possible.
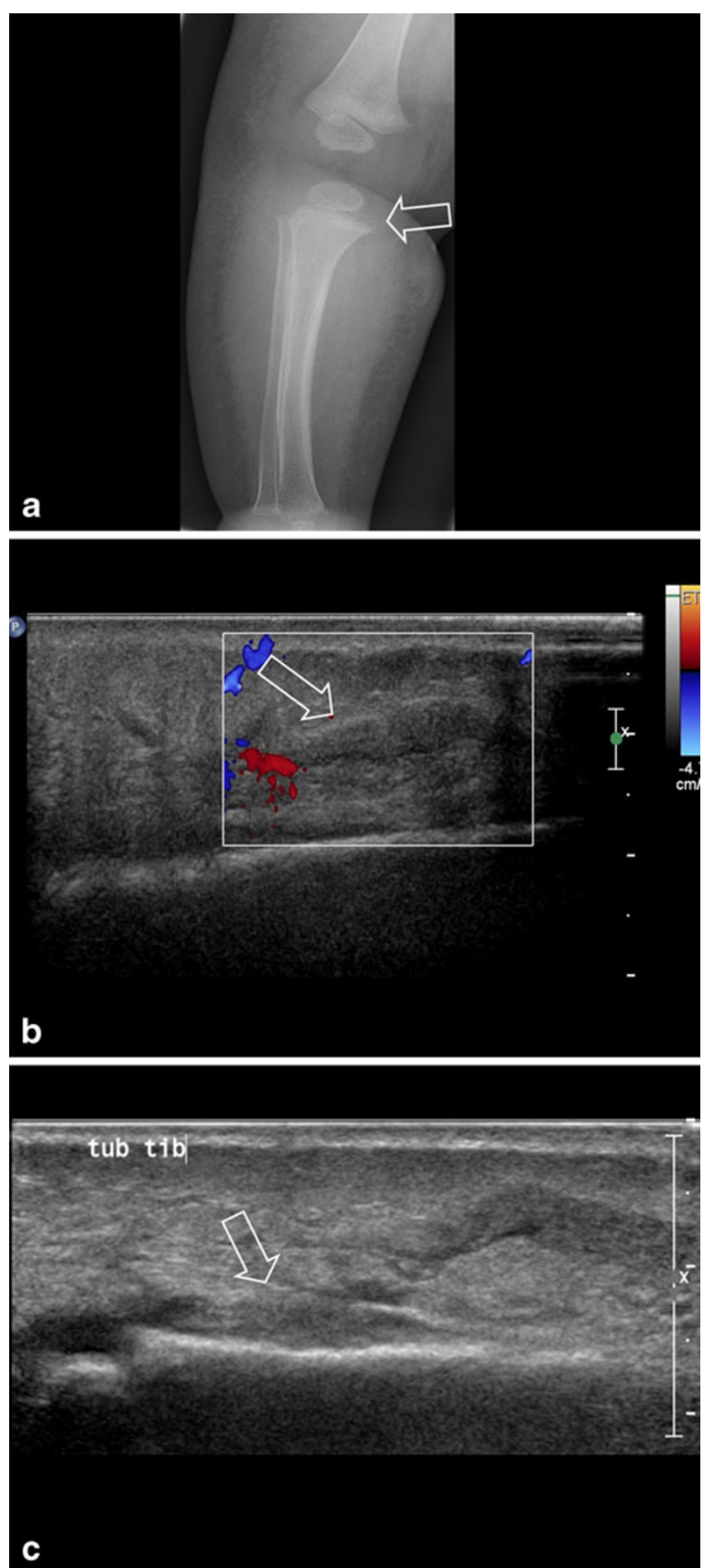

Fig. 5 a A 3-month-old girl with tenderness of the right leg and fever. Imaging shows soft tissue swelling around the tibia. The periostium of the proximal metaphysis is slightly irregular (arrow). b Ultrasound with Doppler shows infiltration of the subcutis, hyperaemia and a long subcutaneous fluid collection (arrow). c Subperiostal fluid collection/ abscess (arrow shows uplifted periostium). Surgically the abscess was drained and the patient received antibiotics 

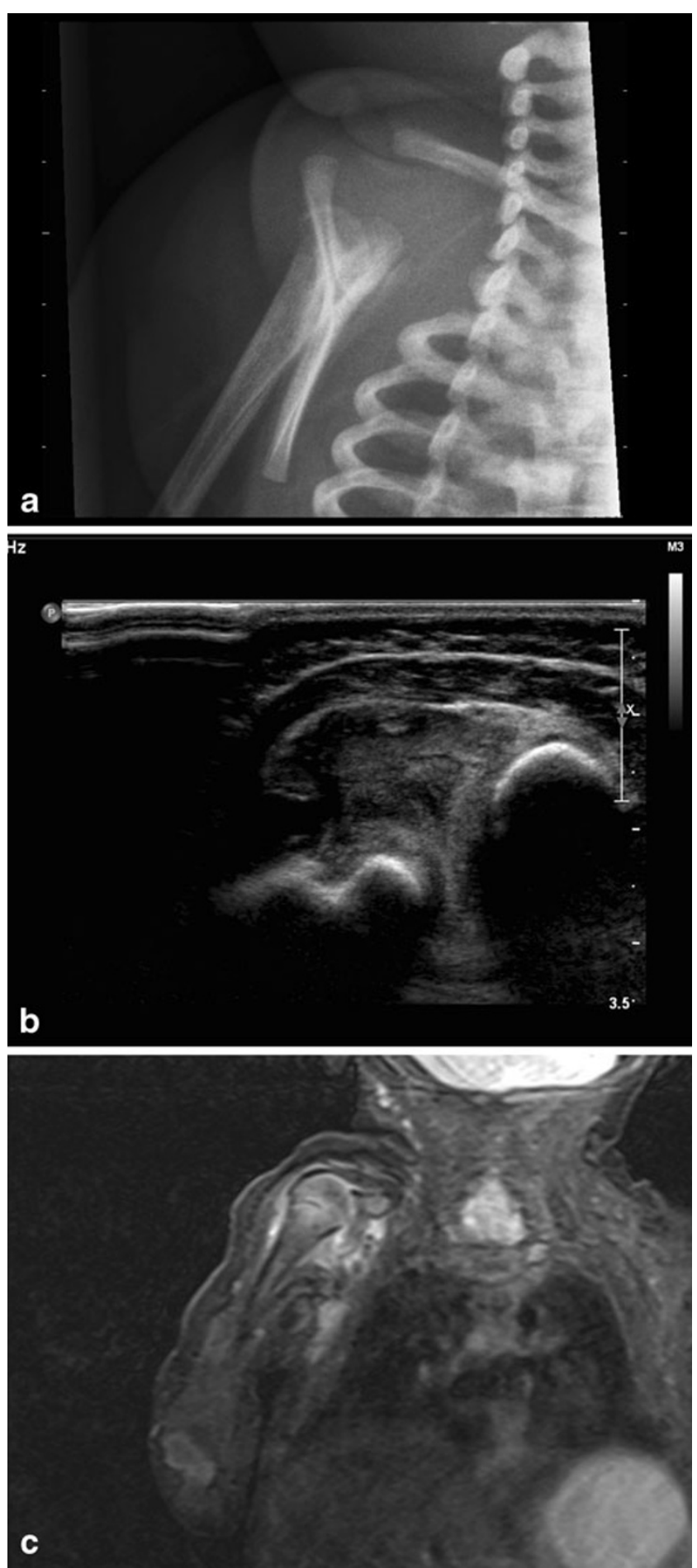

Fig. 6 a A 1.5-month-old girl with low grade fever and reluctance to move her right shoulder. Conventional imaging shows a lucent lesion with possible cortical disruption dorsal in the head of the humerus and periostal reaction in the proximal third of the humerus. b Ultrasound study revealed hyperechoic fluid in the shoulder joint, as seen in septic arthritis. c Coronal T1 STIR series, although with motion artefact, showed high signal around the humeral head and high signal in the bone marrow of the proximal third of the shaft. The final diagnosis was septic arthritis in combination with osteomyelitis of the proximal humerus
In children, compared to adults, the periostium is loosely attached to the bone, and in case of infection it can easily be lifted, thereby creating a space for pus collection.

Multiple foci of osteomyelitis can be seen in children with sickle cell disease, diabetes mellitus and chronic granulomatous disease [20].

\section{Subacute osteomyelitis}

An osteomyelitis with symptoms longer than 2 weeks is defined as subacute osteomyelitis and shows more classical signs on conventional imaging, including single or laminated periostal reactions, and well-circumscribed metaphysical lucency in the long bones, also known as Brodie's abscess (Fig. 4) [20].
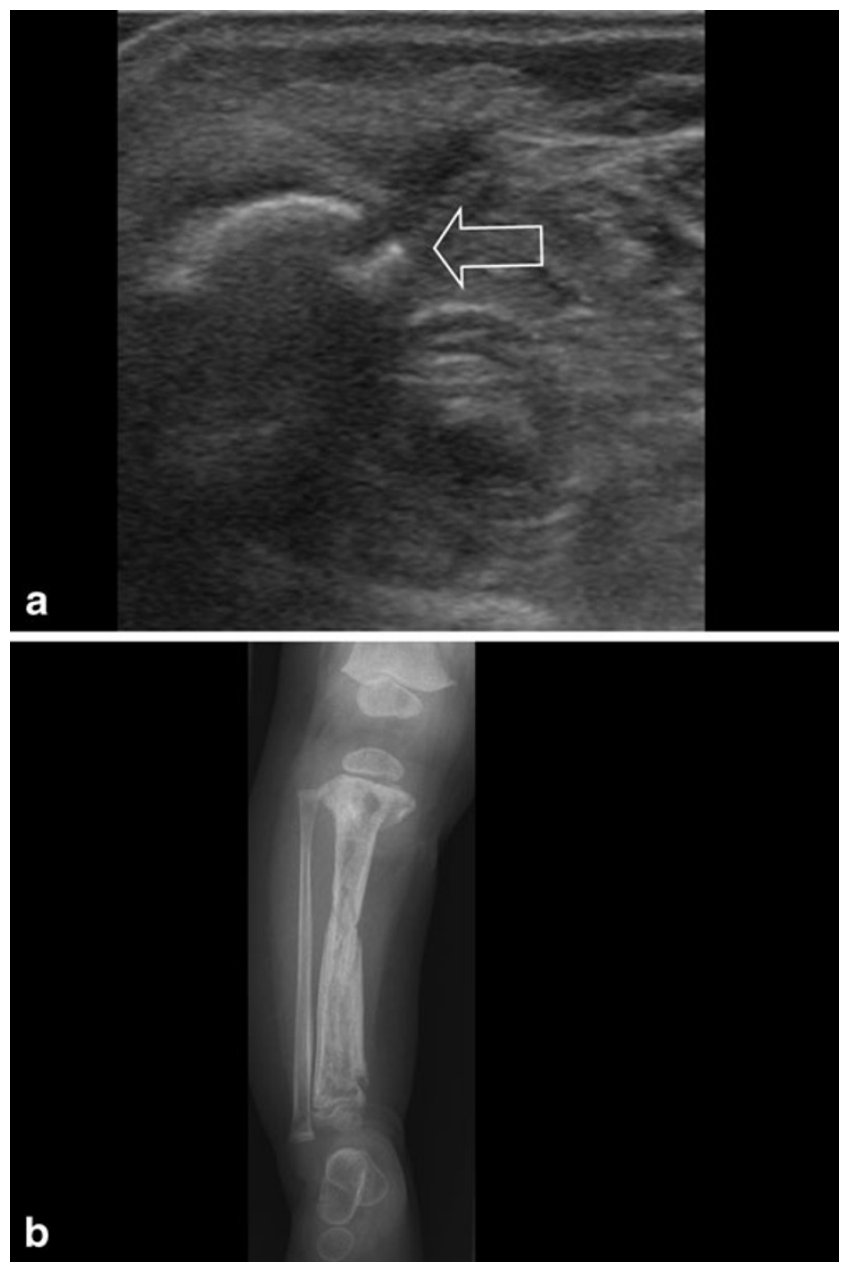

Fig. 7 a Same patient as in Fig. 4 was treated with antibiotics for 1 month. Because of low-grade fever, ultrasonography was repeated. A rest of the abscess was seen as well as extensive cortical destruction at multiple locations (arrow shows one). b Conventional image was performed to evaluate the extensiveness, showing complete involvement of the tibia, with fracture lines in the proximal and distal metaphysis, and mid diaphysis 


\section{Imaging findings}

Conventional imaging In the first $48 \mathrm{~h}$, deep soft tissue swelling in the metaphyseal region and loss of fat planes can be visible on conventional imaging (Fig. 5).

Several days after the onset of symptoms, periostal reactions can be seen (Figs. 6 and 5), and after 7 to 21 days lytic lesions can be seen in affected bones (Figs. 6 and 4). Eventually, this can lead to marked destruction of the bone with extension into the cortex ("endostal scalloping"). Bone destruction can appear as lucency but also in a permeative pattern. Destruction can be limited to the metaphysis, but can also, in rare cases, if misdiagnosed or left untreated, extend into the physis and epiphysis (Fig. 7). This can be very difficult to diagnose on conventional radiographs because in children the epiphysis is not yet ossified $[3,4,6,19,26]$. The presence of a joint
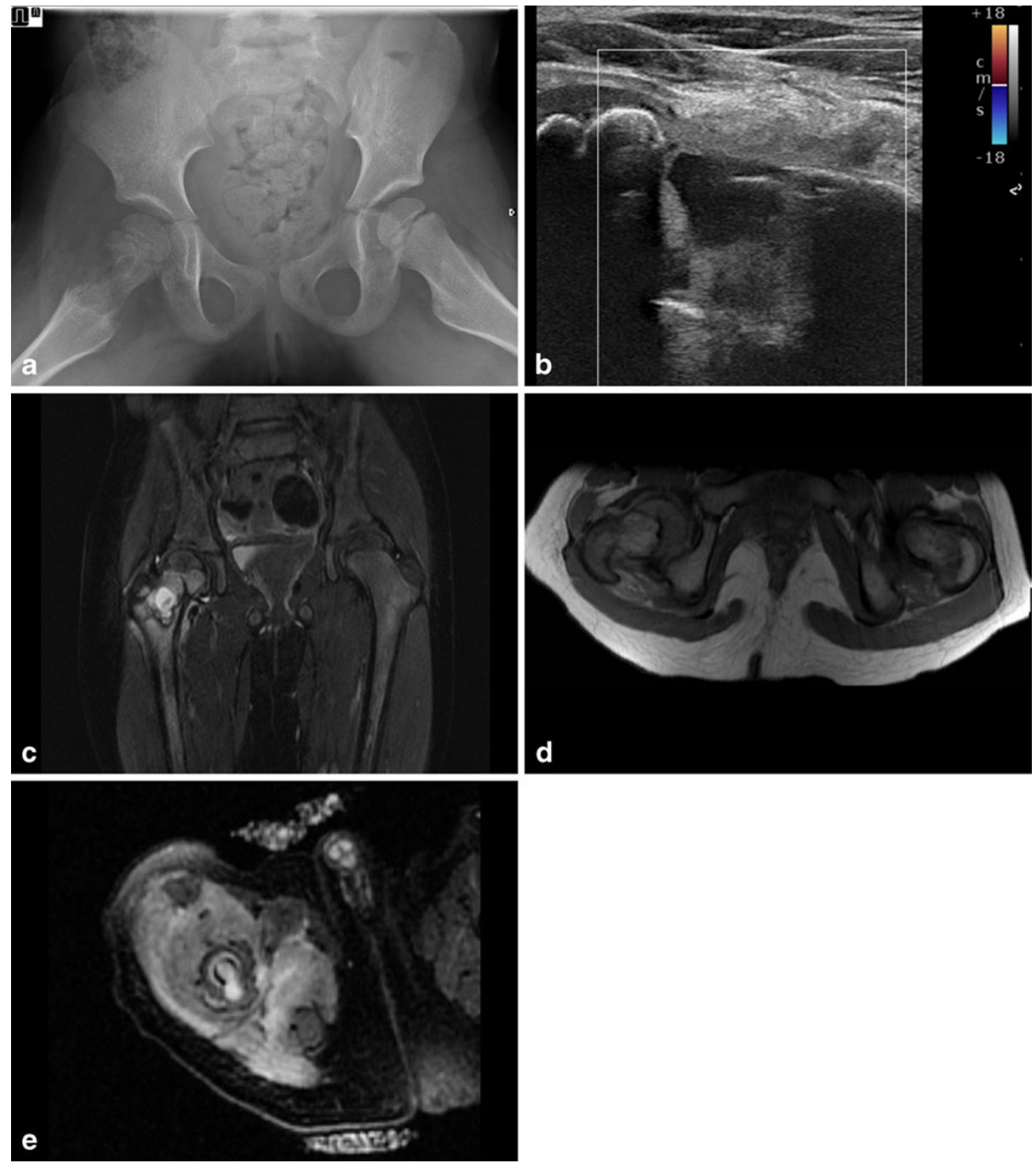

Fig. 8 a A 3-month-old boy with a fever and reluctance to use his right limb. Physical examination shows a swollen, red hip. Conventional imaging shows extensive destruction of the proximal right femur and swollen soft tissue. b Ultrasound of the right hip shows cortical destruction, the formation of a subperiostal fluid collection/abscess and infiltration of soft tissue. c-d Coronal T1 STIR and axial PD images show cortical destruction and fluid collection/abscess formation in bone marrow. No contrast-enhanced imaging was performed in this case. e Axial T2 FS image confirms the fluid/abscess collection with extension outside the bone 
effusion can be a helpful clue, although plain radiographs have a low sensitivity for detecting joint effusion (Fig. 8).

Ultrasound US allows evaluation of soft tissue swelling due to oedema or fluid collection, and hyperaemia can be detected using Doppler techniques. Also periosteal thickening and sub-periostal collections are seen. These findings can precede abnormalities on conventional imaging in the first week (Figs. 5 and 7).

US has high sensitivity for detection of intra-articular fluid $[3,4,21,24,25,30]$ (Figs. 6 and 8).

In more extensive cases, cortical defects can also be detected (Fig. 8). It can be useful for detecting foreign bodies, especially non-radioopaque foreign bodies such as wood splinters as a causative agent. Sometimes it is possible to image formation of a sequesters, especially when surrounded by pus (Fig. 9).
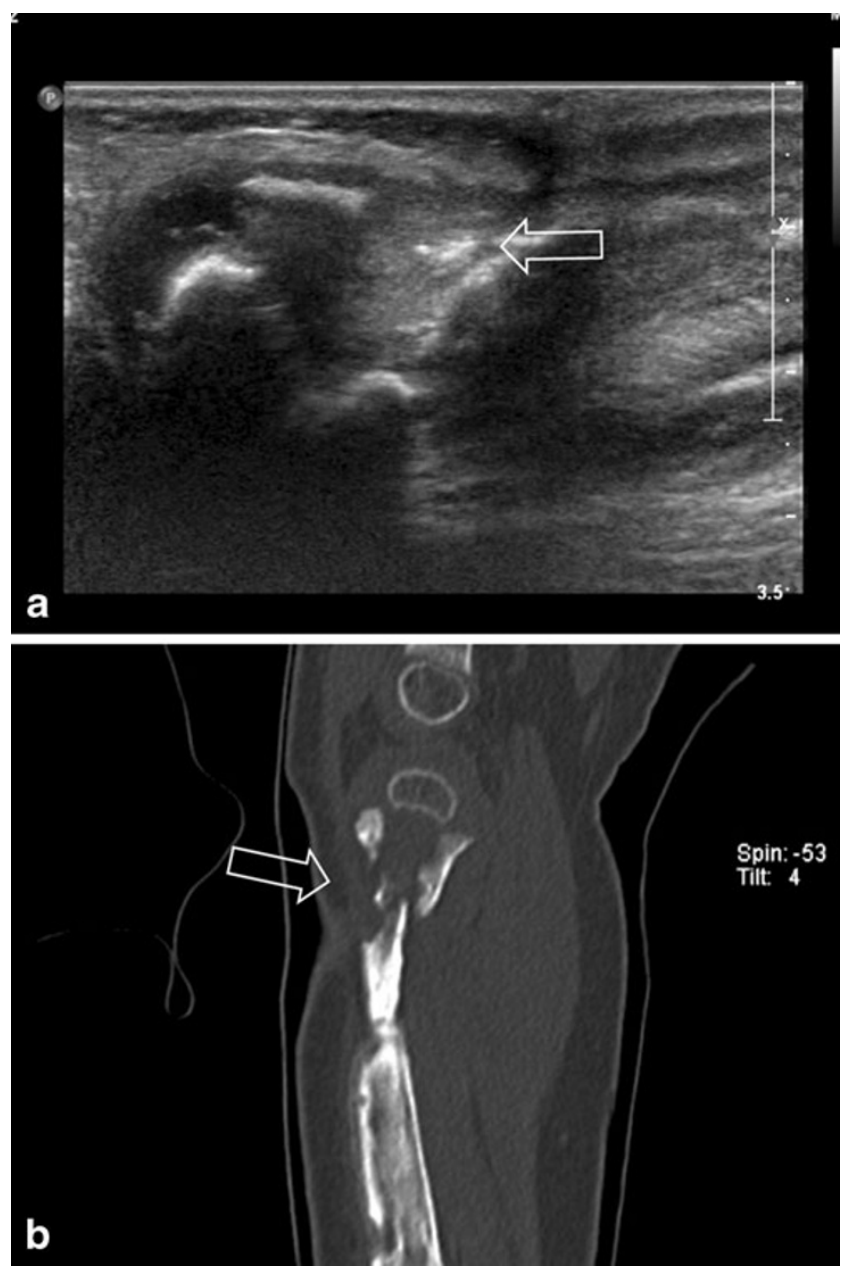

Fig. 9 a A 3-month-old girl after treatment for osteomyelitis. In the follow-up, there was the suspicion of a sequester in the proximal metaphysis. Ultrasound was performed, which showed hyperechoic fragments in a surrounding fluid collection, suspicious for a small sequester (arrow). b CT confirmed the presence of a small sequester (arrow)
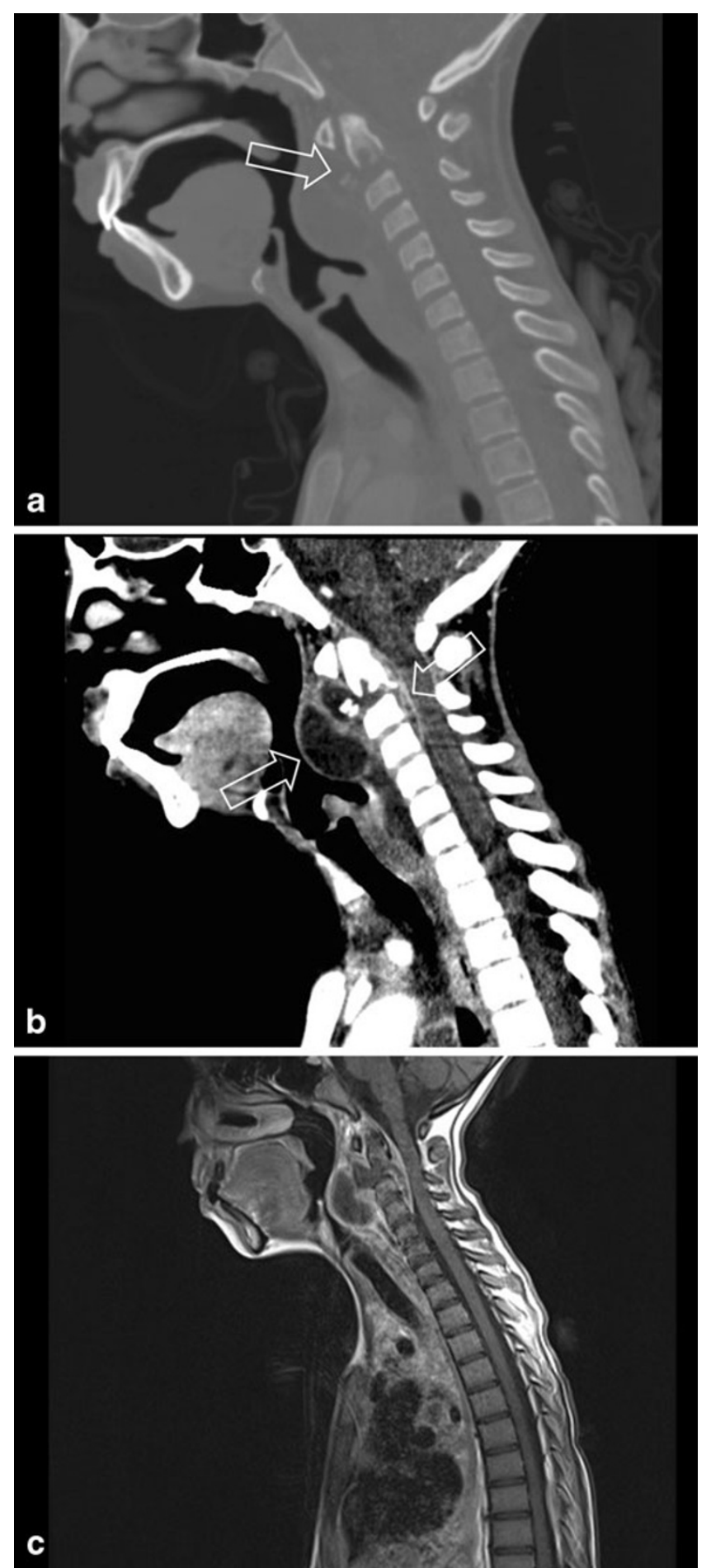

Fig. 10 a A 9-year-old girl from Ethiopia, with cervical lymph nodes and weight loss, under suspicion of having tuberculosis. Fluid collection was seen on ultrasound at level II and the patient underwent CT to exclude a retropharyngeal abscess. CT showed multiple cervical and mediastinal lymph nodes, but also destruction of the end plate of the corpus of $\mathrm{C} 2$ with a fragment dislocated anteriorly (arrow). b CT in the soft tissue kernel clearly shows a prevertebral fluid collection with rim enhancement, but also posterior extension in the spinal canal, with enhancement. The diagnosis is a tuberculous spondylodiscitis. c On the sagittal T1 contrastenhanced fat-saturated image, one can clearly see the anterior abscess, but also the intraspinal extension of the abscess in the spinal canal 
Computed tomography $\mathrm{CT}$ is useful in the evaluation of bony destruction (Fig. 10) and in cases with complications such as abscess, fistula or sequester formation (Fig. 9). Intravenous contrast agents can be useful for the evaluation of soft tissue extension, although MRI has a higher sensitivity for the evaluation of soft tissue involvement (Fig. 10).

Magnetic resonance imaging After 3-5 days of onset of infection, MRI can detect osteomyelitis [35]. MRI is the best technique available to evaluate changes in the bone marrow/water content of bone marrow. Alteration of bone marrow signal intensity can be visible 1 or 2 days after the onset of infection (Figs. 6 and 8) [3].

MRI criteria for diagnosis are low signal intensity on T1, high signal intensity on T2/STIR, and enhancement of (subperiosteal) bone and/or soft tissues and abscess/collections after contrast administration [36].

MRI is also useful for evaluation of complications such as (subperiosteal) abscesses, joint effusions and soft-tissue extension that would require surgical treatment (Figs. 8, 10 and 11) [19].
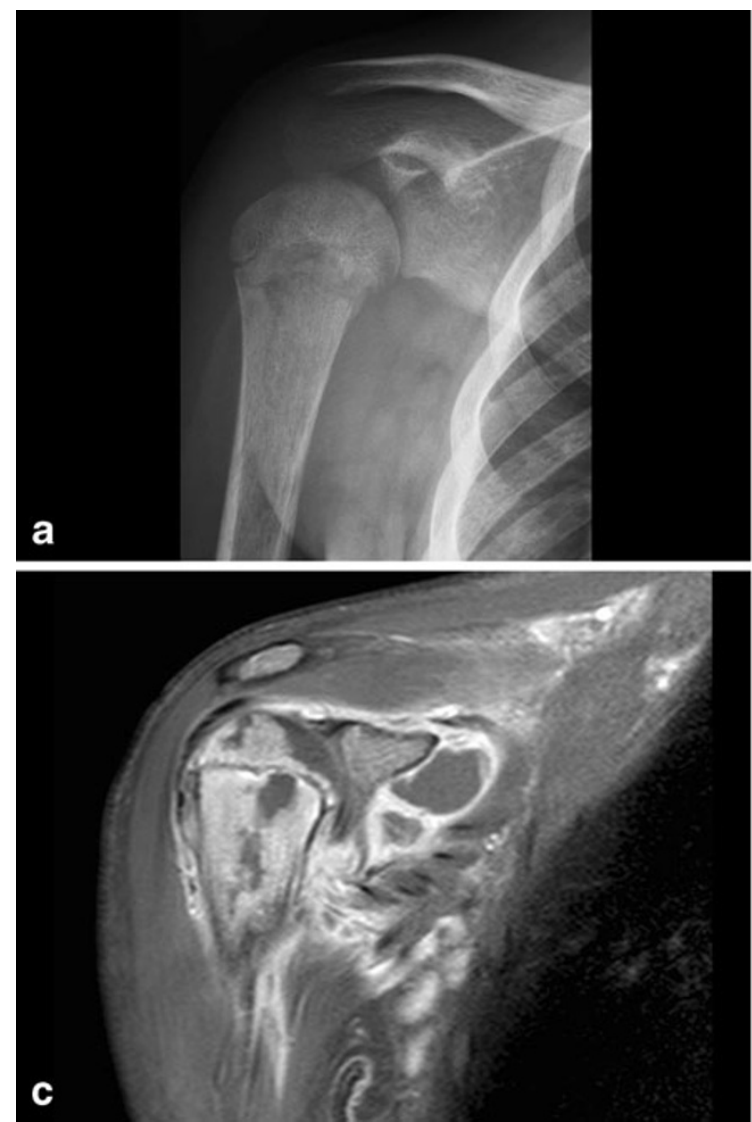

Fig. 11 a A 10-year-old girl, known to have sickle cell disease, with shoulder complaints and high fever. Conventional imaging showed lucency in the humeral head and periostal reaction. b-c Axial, sagittal T1 FS contrast-enhanced series shows extensive abscess formation in
Nuclear imaging Acute osteomyelitis on a three-phase bone scintigraphy can show increased blood flow and blood pool activity, and positive uptake on $3 \mathrm{~h}$ images in areas of osteomyelitis. However, at this time blood pool flow and pool activity can be normal or photon deficient because of devascularisation of the periostium due to rapid raising of the periostium [4, 37].

PET-CT will show high uptake in areas of osteomyelitis, in bone marrow and in soft tissue.

Differential diagnosis (Table 2) Since defining a differential diagnosis solely based on imaging characteristics can be very difficult, clinical and laboratory parameters and communication with clinicians remain very important in the diagnosis of osteomyelitis. In cases of doubt, fine-needle aspiration or an image-guided biopsy will eventually be necessary to determine the definitive diagnosis.

Patients without infectious parameters In patients without any infectious parameters, the differential diagnosis is mainly based on disease of vascular origin, neoplasm or trauma.
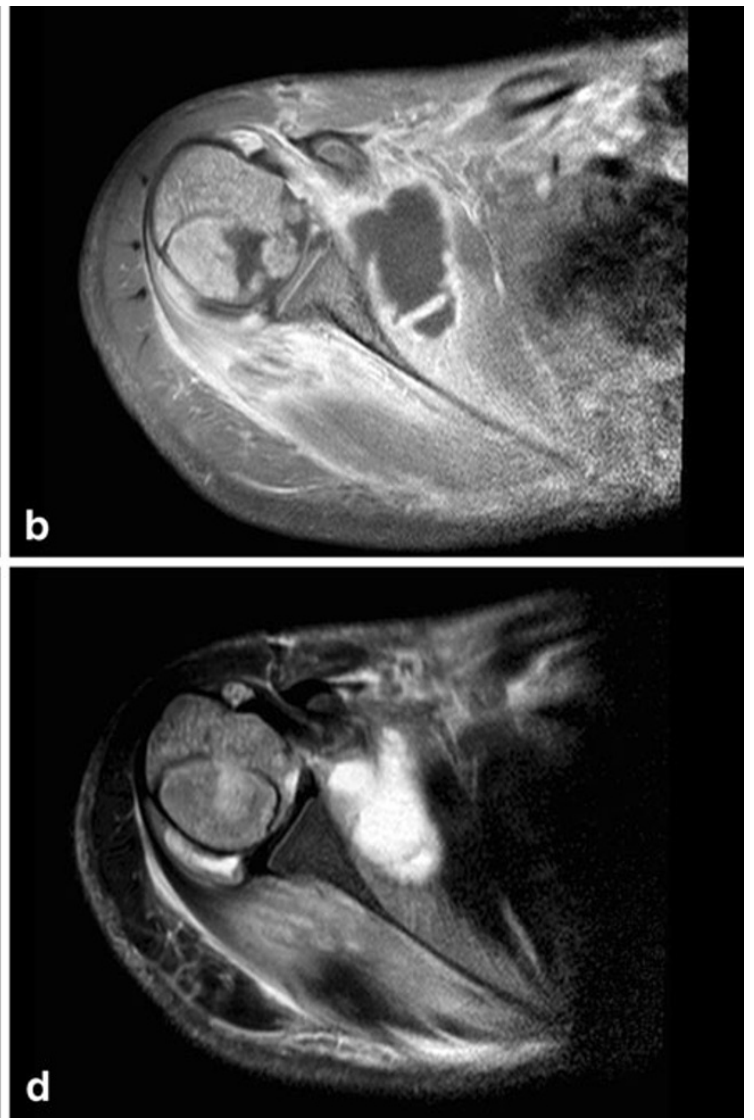

the subscapular region and irregular T1 low signal intensity bone lesions. d Axial T2 FS shows extensive infiltration of soft tissue, abscess and also a joint effusion. This turned out to be caused by salmonella infection 


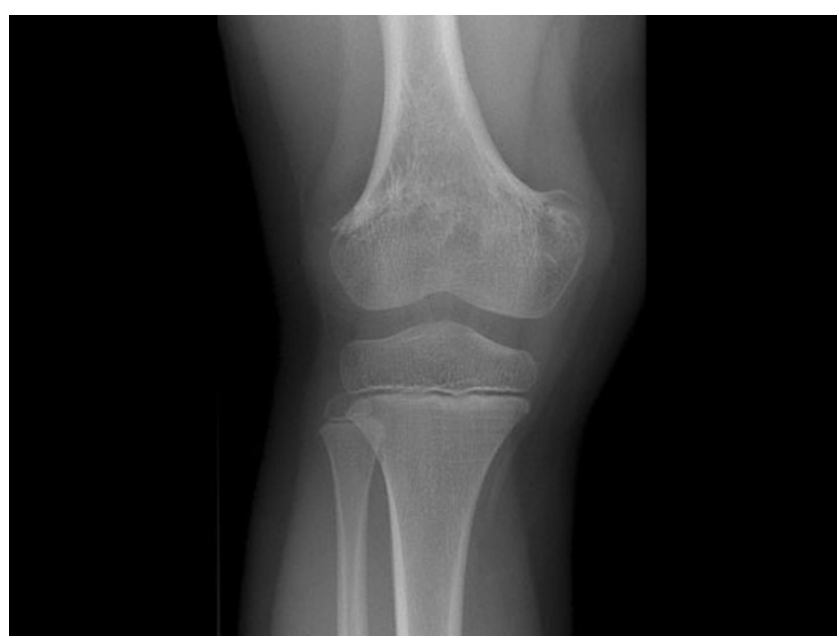

Fig. 12 Premature closure of the epiphysis of the right femur after osteomyelitis

This group without infectious parameters can be the most challenging for the differential diagnosis.

Patients with a history of use of medications such as corticosteriods or for example patients with sickle cell disease must
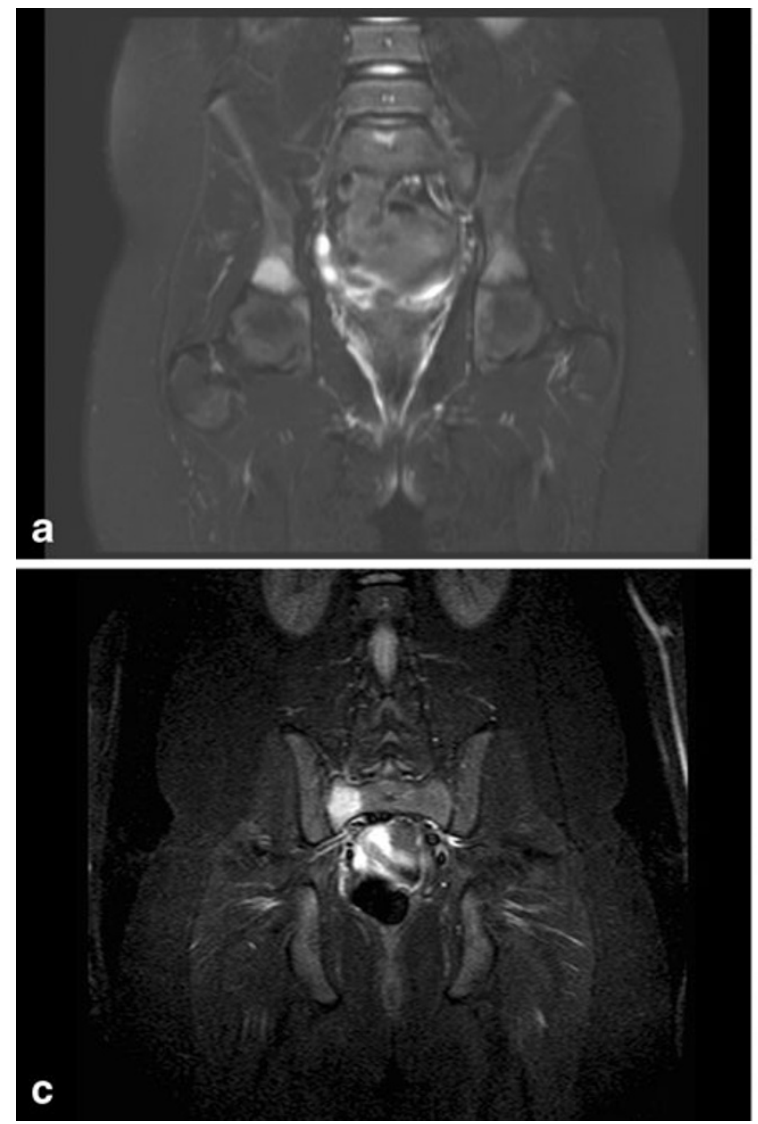

Fig. 13 a-b An 11-year-old girl with pain in her loin. Conventional imaging did not show any abnormalities. T2 SPIR imaging showed multiple areas of high signal in both the acetabulum and the neck of the left femur. No contrast was given. Diagnosis was CRMO. c-d STIR raise the suspicion of vaso-occlusive disease. Imaging will show typical serpentinous sclerotic lesions on conventional imaging, whereas MR imaging will show linear hypointense T1- and T2-weighted changes in the meta- and epiphysis [26].

Ewing's sarcoma produces a large soft tissue mass without calcifications or bone matrix in the tissue. Onion-skin periostitis on conventional imaging is typical.

Osteosarcoma shows a lesion usually in the diaphysis with signs of aggressive (cortical) bone destruction and periostic reactions such as Codman's triangle and sunburst spiculated periostitis.

In a patient with a history of trauma, stress fractures will show linear, hypointense changes on T1-weighted imaging, without enhancement. Bone marrow oedema can be visible on STIR imaging.

When imaging shows an osteolytic lesion with a central nidus surrounded by a sclerotic margin in a patient with a typical history of night pain relieved by use of NSAIDs, one should consider the diagnosis of osteoid osteoma.

In patients with suspicion of haematologic malignancy such as ALL, diffuse infiltration of the bone marrow can be seen. Imaging will show diminished signal intensities on T1
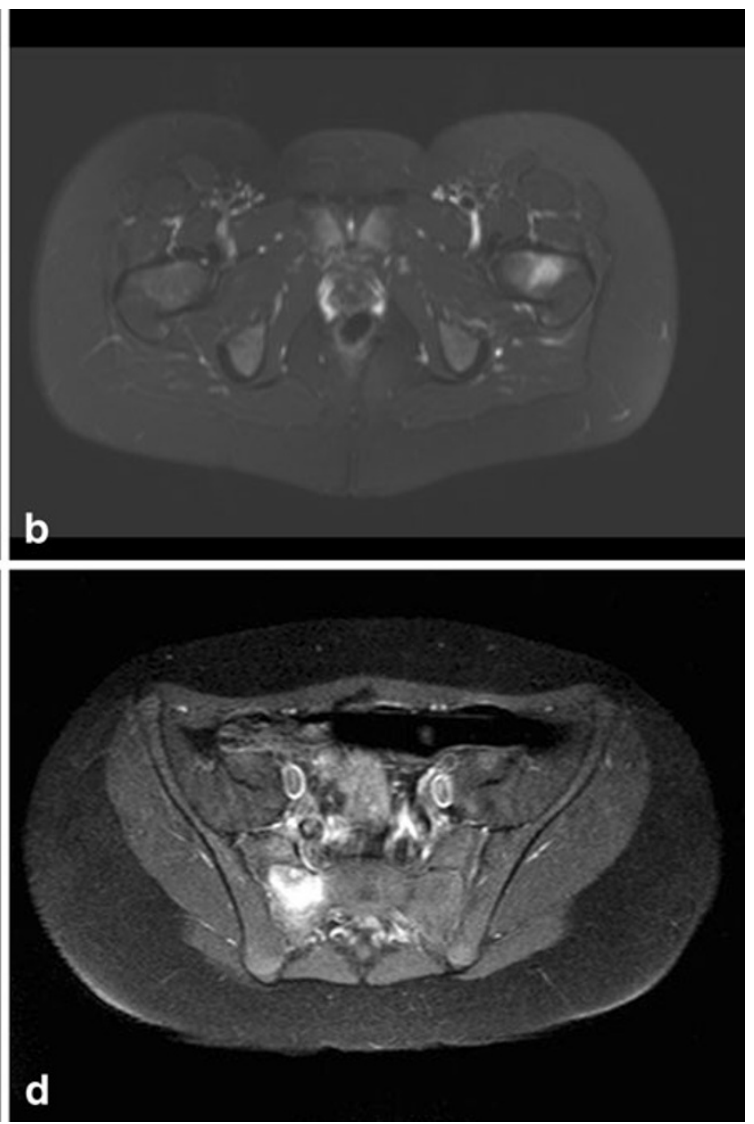

TSE image obtained 2 months later shows only high signal intensity in the os sacrum on the right, without joint effusion of the sacroiliac joint. No other lesions were seen. T1 FS TSE contrast-enhanced series showed enhancement of the lesion 
imaging; T2 will show heterogenic signals. Metastastic neuroblastoma can show multiple lesions usually characterised only by high signal intensity on STIR imaging.

In young children with a red painful or asymptomatic lesion on the sternum with or without infectious parameters, one should consider a self-limiting sternal tumour of childhood (SELSTOC) [38]. Ultrasound in these patients will show a dumbbell-shaped lesion extending to the area behind the sternal bone. No additional imaging will be needed in these patients.

Patients with or after a period with infectious parameters In patients with infectious parameters in combination with backache, one must consider a spondylodiscitis. Imaging shows low signal of the disc with surrounding fluid/abscess with destruction of the vertebrae and rim enhancement after gadolinium administration (Fig. 10); Pott's disease in tuberculosis will be more pronounced in the facet joints.

After a period of septicaemia, such as a fulminant meningococcemia, septic emboli can occur. This can lead to partial premature closure of the physis leading to joint deformities (Fig. 12).
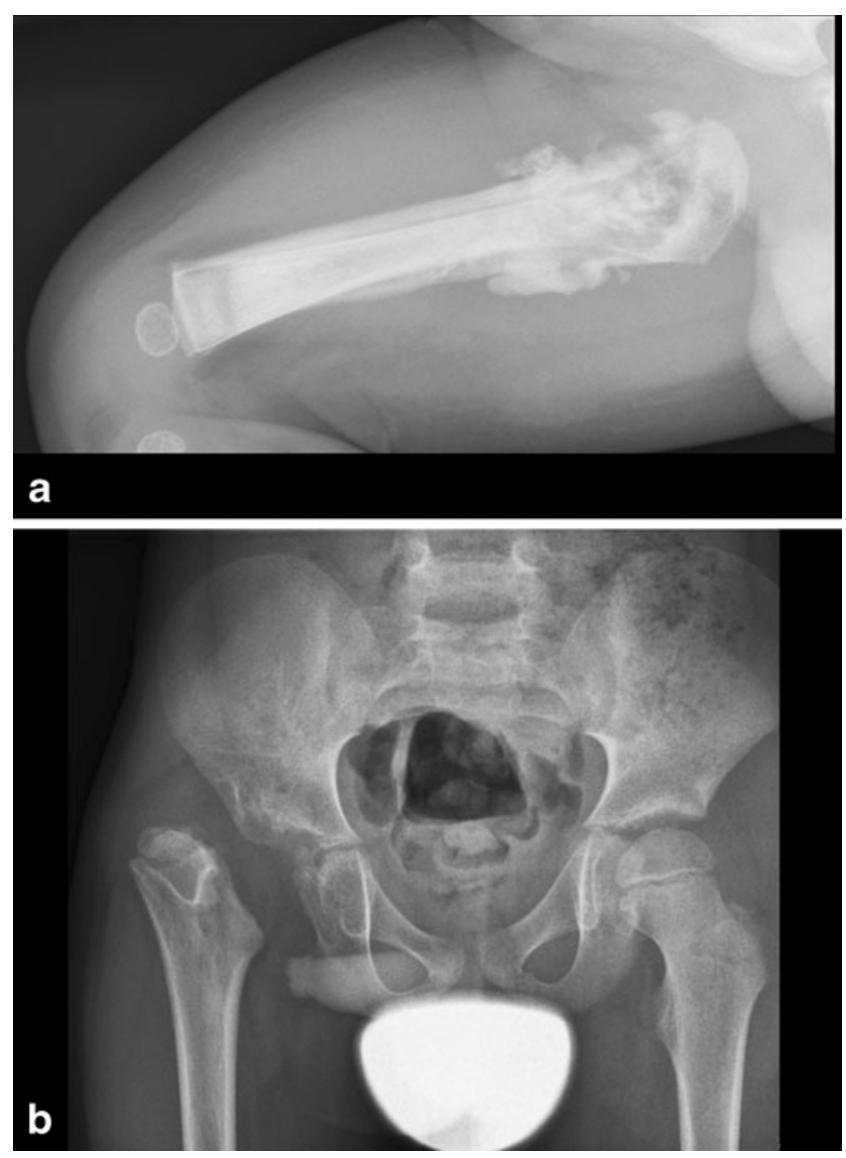

Fig. 14 a A 6-week-old boy with fever. X-ray shows extensive cortical destruction of the proximal metaphysis with surrounding soft tissue calcifications and periostal reaction. This was proven to be septic osteoarthritis. b At age 7 years X-ray shows (acquired) deformity of the caput femoris, with luxation

\section{Chronic osteomyelitis}

Chronic osteomyelitis is defined as a persistent or recurrent low-grade bone infection. Usually this occurs in older patients after trauma or surgery.

Conventional imaging Conventional imaging will show the same features as in acute osteomyelitis, although more extensively (Fig. 7). Complications such as fractures or sequester formation can be seen.

Computed tomography CT plays an important role in the diagnosis and analysis of chronic osteomyelitis. Imaging of complications of osteomyelitis such intraosseous abscesses, sequesters, and fistulas or cloaca formation is important (Figs. 8 and 9).

Soft tissue extension can be evaluated, but to a lesser extent compared to MR. Imaging characteristics are comparable to those for acute osteomyelitis.

Magnetic resonance imaging MR can show soft tissue extension and abscess. Also intraosseous abscesses and fistulas can be seen. Imaging characteristics are comparable to those of acute osteomyelitis. Use of total body STIR can exclude multifocal disease.

PET PET scans will show uptake in the area of osteomyelitis. It can exclude multifocality.

Differential diagnosis (Table 2) The main differential diagnosis of chronic osteomyelitis consists of neoplasms.

In Ewing's sarcoma a large soft tissue mass without calcifications or bone matrix in the tissue can be seen. Onion-skin periostitis is typical.

$\mathrm{LCH}$ can be difficult to differentiate from multifocal osteomyelitis. LCH typically produces punched-out lesions on conventional imaging. Therefore, conventional imaging can provide a valuable clue.

Bone metastases are usually multifocal. In this diagnosis also the clinical parameters are important signs; in most cases, there are no signs of inflammation.

Chronic recurrent multifocal osteomyelitis (CRMO) can be part of SAPHO syndrome (synovitis, acne, pustulosis, hyperostosis, osteitis). It is a very uncommon condition, of unknown aetiology, with a peak incidence between the ages of 4-14 years. Characteristically, there are multiple locations, but only one is symptomatic [39]. The imaging strategy usually has a symptomatic focus, as in acute osteomyelitis, but multiple lesions will be shown. Especially MR and PET can be used to analyse multiple lesions. STIR and T2 series show multiple spots of high signal intensity and series after contrast show enhancement. Imaging characteristics are comparable to those of 
acute osteomyelitis. The focus of osteomyelitis and symptoms can change over time (Fig. 13).

\section{Follow-up}

To date no data have been published on the use of follow-up imaging in childhood osteomyelitis. In a retrospective study by Kowalski et al. on the use of follow-up MRI for the prognosis of spinal osteomyelitis, they found that the most valid parameter on follow-up MRI was a change in soft tissue involvement. The authors concluded that only in selected cases were MR findings of additional value [40].

Only data of small-group studies on follow-up of osteomyelitis with bone scintigraphy and FDG-PET are available. These studies suggest that in the follow-up of osteomyelitis, FDG-PET and bone scintigraphy can be useful to analyse the activity of osteomyelitis and that FDG-PET is superior to MRI. Activity can be a parameter to determine the time for termination of therapy $[19,41]$.

Unique to childhood are the late complications of osteomyelitis due to the growth and developmental disturbances of the affected bone. These developmental changes can involve malformations of the joints (Figs. 12 and 14), leading to e.g. contractures or loss of functionality, or whole segments of bone, e.g. non-development as a result of osteonecrosis. These possible serious long-term consequences of childhood osteomyelitis indicate that these children should undergo clinical and radiological follow-up at some point in time.

\section{Conclusion}

Osteomyelitis in childhood can be a challenging diagnosis. Imaging plays a vital role in the diagnosis of childhood osteomyelitis, and the imaging findings are pivotal in the treatment decision. As in all clinical cases the most important aspect of the diagnosis of childhood osteomyelitis lies in good cooperation among the paediatrician, paediatric orthopaedic surgeon and paediatric radiologist.

Open Access This article is distributed under the terms of the Creative Commons Attribution License which permits any use, distribution, and reproduction in any medium, provided the original author(s) and the source are credited.

\section{References}

1. Medina LS, Applegate KE, Blackmore C (2010) Evidence-Based Imaging in Pediatrics

2. Kao HC, Huang YC, Chiu CH, Chang LY, Lee ZL, Chung PW, Kao FC, Lin TY (2003) Acute hematogenous osteomyelitis and septic arthritis in children. J Microbiol Immunol Infect 36:260-265
3. Pineda C, Vargas A, Rodriguez AV (2006) Imaging of osteomyelitis: current concepts. Infect Dis Clin N Am 20:789-825

4. Jaramillo D (2011) Infection: musculoskeletal. Pediatr Radiol 41 (Suppl 1):S127-S134

5. Dahl LB, Hoyland AL, Dramsdahl H, Kaaresen PI (1998) Acute osteomyelitis in children: a population-based retrospective study 1965 to 1994 . Scand J Infect Dis 30:573-577

6. Riise OR, Kirkhus E, Handeland KS, Flato B, Reiseter T, Cvancarova M, Nakstad B, Wathne KO (2008) Childhood osteomyelitis-incidence and differentiation from other acute onset musculoskeletal features in a population-based study. BMC Pediatr 8:45

7. www.Uptodate.com Pediatric Osteomyelitis. 2011.

8. Espinosa CM, Davis MM, Gilsdorf JR (2011) Anaerobic osteomyelitis in children. Pediatr Infect Dis J 30:422-423

9. Steer AC, Carapetis JR (2004) Acute hematogenous osteomyelitis in children: recognition and management. Paediatr Drugs 6:333-346

10. Gafur OA, Copley LA, Hollmig ST, Browne RH, Thornton LA, Crawford SE (2008) The impact of the current epidemiology of pediatric musculoskeletal infection on evaluation and treatment guidelines. J Pediatr Orthop 28:777-785

11. A. G. Crossley KB (1997) The staphylococci in human disease. Churchill Livingstone

12. Teo HE, Peh WC (2004) Skeletal tuberculosis in children. Pediatr Radiol 34:853-860

13. Howard AW, Viskontas D, Sabbagh C (1999) Reduction in osteomyelitis and septic arthritis related to Haemophilus influenzae type B vaccination. J Pediatr Orthop 19:705-709

14. Jain R, Sawhney S, Rizvi SG (2008) Acute bone crises in sickle cell disease: the T1 fat-saturated sequence in differentiation of acute bone infarcts from acute osteomyelitis. Clin Radiol 63:5970

15. Gotthardt M, Bleeker-Rovers CP, Boerman OC, Oyen WJ (2010) Imaging of inflammation by PET, conventional scintigraphy, and other imaging techniques. J Nucl Med 51:1937-1949

16. Goergens ED, McEvoy A, Watson M, Barrett IR (2005) Acute osteomyelitis and septic arthritis in children. J Paediatr Child Health 41:59-62

17. Karwowska A, Davies HD, Jadavji T (1998) Epidemiology and outcome of osteomyelitis in the era of sequential intravenous-oral therapy. Pediatr Infect Dis J 17:1021-1026

18. Le SN, Howard A, Barrowman NJ, Gaboury I, Sampson M, Moher D (2002) Shorter courses of parenteral antibiotic therapy do not appear to influence response rates for children with acute hematogenous osteomyelitis: a systematic review. BMC Infect Dis 2:16

19. Karmazyn B, Kim JY, Jaramillo D (2010) Imaging of hematogenous osteomyelitis and septic arthritis in children. In: Medina LS, Applegate KE, Blackmore CC (eds) Evidence-based imaging in pediatrics. Springer, pp. 245-258

20. Blickman JG, Vanderschueren G (2009) Skeletal system. In: Blickman JG, Parker BR, Barnes PD (eds) Pediatric radiology. Mosby Elsevier, The Requisites, pp 157-205

21. Robben SG (2004) Ultrasonography of musculoskeletal infections in children. Eur Radiol 14(Suppl 4):L65-L77

22. Zamzam MM (2006) The role of ultrasound in differentiating septic arthritis from transient synovitis of the hip in children. $\mathrm{J}$ Pediatr Orthop B 15:418-422

23. Azam Q, Ahmad I, Abbas M, Syed A, Haque F (2005) Ultrasound and colour Doppler sonography in acute osteomyelitis in children. Acta Orthop Belg 71:590-596

24. Karmazyn B (2010) Imaging approach to acute hematogenous osteomyelitis in children: an update. Semin Ultrasound CT MR 31:100-106

25. Pineda C, Espinosa R, Pena A (2009) Radiographic imaging in osteomyelitis: the role of plain radiography, computed tomography, ultrasonography, magnetic resonance imaging, and scintigraphy. Semin Plast Surg 23:80-89 
26. Schmit P, Glorion C (2004) Osteomyelitis in infants and children. Eur Radiol 14(Suppl 4):L44-L54

27. Shah NB, Platt SL (2008) ALARA: is there a cause for alarm? Reducing radiation risks from computed tomography scanning in children. Curr Opin Pediatr 20:243-247

28. Termaat MF, Raijmakers PG, Scholten HJ, Bakker FC, Patka P, Haarman HJ (2005) The accuracy of diagnostic imaging for the assessment of chronic osteomyelitis: a systematic review and metaanalysis. J Bone Joint Surg Am 87:2464-2471

29. Taghon TA, Bryan YF, Kurth CD (2006) Pediatric radiology sedation and anesthesia. Int Anesthesiol Clin 44:65-79

30. Jaramillo D, Laor T (2008) Pediatric musculoskeletal MRI: basic principles to optimize success. Pediatr Radiol 38:379-391

31. Kan JH, Young RS, Yu C, Hernanz-Schulman M (2010) Clinical impact of gadolinium in the MRI diagnosis of musculoskeletal infection in children. Pediatr Radiol 40:1197-1205

32. Dipoce J, Jbara ME, Brenner AI (2012) Pediatric osteomyelitis: a scintigraphic case-based review. Radiographics 32:865-878

33. van der Bruggen W, Bleeker-Rovers CP, Boerman OC, Gotthardt M, Oyen WJ (2010) PET and SPECT in osteomyelitis and prosthetic bone and joint infections: a systematic review. Semin Nucl Med 40:3-15

34. IAEA radiation protection of patients. Online publication 2011

35. Kocher MS, Lee B, Dolan M, Weinberg J, Shulman ST (2006) Pediatric orthopedic infections: early detection and treatment. Pediatr Ann 35:112-122
36. Offiah AC (2006) Acute osteomyelitis, septic arthritis and discitis: differences between neonates and older children. Eur J Radiol 60:221-232

37. Connolly LP, Connolly SA, Drubach LA, Jaramillo D, Treves ST (2002) Acute hematogenous osteomyelitis of children: assessment of skeletal scintigraphy-based diagnosis in the era of MRI. J Nucl Med 43:1310-1316

38. te Winkel ML, Lequin MH, de Bruyn JR, van de Ven CP, de Krijger RR, Pieters R, van den Heuvel-Eibrink MM (2010) Selflimiting sternal tumors of childhood (SELSTOC). Pediatr Blood Cancer 55:81-84

39. Tlougan BE, Podjasek JO, O'Haver J, Cordova KB, Nguyen XH, Tee R, Pinckard-Hansen KC, Hansen RC (2009) Chronic recurrent multifocal osteomyelitis (CRMO) and synovitis, acne, pustulosis, hyperostosis, and osteitis (SAPHO) syndrome with associated neutrophilic dermatoses: a report of seven cases and review of the literature. Pediatr Dermatol 26:497-505

40. Kowalski TJ, Layton KF, Berbari EF, Steckelberg JM, Huddleston PM, Wald JT, Osmon DR (2007) Follow-up MR imaging in patients with pyogenic spine infections: lack of correlation with clinical features. AJNR Am J Neuroradiol 28:693-699

41. Warmann SW, Dittmann H, Seitz G, Bares R, Fuchs J, Schafer JF (2011) Follow-up of acute osteomyelitis in children: the possible role of PET/CT in selected cases. J Pediatr Surg 46: $1550-1556$ 